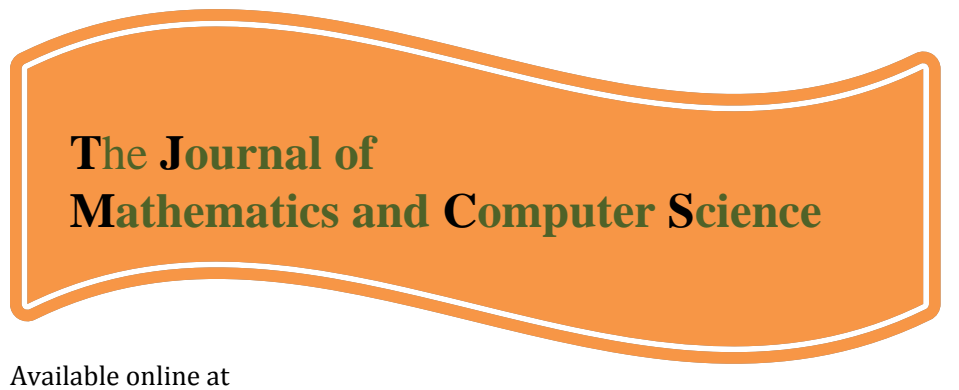

http://www.TJMCS.com

The Journal of Mathematics and Computer Science Vol .2 No.2 (2011) 255-261

\title{
Bidirectional Image Thresholding algorithm using combined Edge Detection and $P$-Tile algorithms
}

\author{
Moslem Taghizadeh ${ }^{1,}$, Mohammad Reza Mahzoun ${ }^{2}$ \\ Imam Hossein University, Taghizadeh.uni@gmail.com \\ Imam Hossein University, rezamahzoun@yahoo.com
}

Received: September 2010, Revised: November 2010

Online Publication: January 2011

\begin{abstract}
The main disadvantage of traditional global thresholding techniques is that they do not have an ability to exploit information of the characteristics of target images that they threshold. In this paper, we propose a new approach based on combination of modified p-tile and edge detection algorithms to have more accurate object segmentation. Using our proposed method, it is shown that almost all of our experiments resulted to better object segmentation than using traditional methods.
\end{abstract}

Keywords: Image segmentation, Edge detection, P-tile algorithm.

\section{Introduction}

In many applications of image processing, pixel values belonging to the object are substantially different from those in its background. Thresholding is one of the simplest and most commonly used techniques to separate the foreground from its background [1][2][3]. Thresholding techniques can be categorized into two classes: global thresholding and local (adaptive) thresholding. In the global thresholding, a single threshold value is used in the whole image. In the local thresholding, a threshold value is assigned to each pixel to determine whether it belongs to the foreground or the background pixel using local information around the pixel. Because of the advantage of simple and easy implementation,

1, ${ }^{*}$ Corresponding author: M.Sc student in Department of Electronic engineering, Imam Hossein University, Tehran,Iran.

2 PhD in Department of Electronic engineering, Faculty of Engineering, Imam Hossein University, Tehran,Iran. 
the global thresholding has been a popular technique in many years. Techniques based on entropy measures [1][5][6][7] and fuzzy approaches [2][8] have also been proposed. The main disadvantage of traditional thresholding techniques is that they do not have an ability to exploit information of the characteristics of the images that they threshold. They treat all images in the same way, regardless of the specific nature of the images. For some situations, this "one-fits-all" approach is sufficient. However, when greater accuracy and more consistent performance are required, more information should be used to assist the thresholding process.

This paper proposes a method of utilizing shape information to assist thresholding process. We combine the bidirectional P-tile global thresholding method with some edge detection methods to retrieve shape information for assistance, and demonstrate its usefulness in various situations. This is a promising approach because it generates more accurate threshold images than conventional methods especially for applications that need to extract the object shape.

\section{P-tile Thresholding Method}

P-tile tile is a shorter form of the word "percentile". The threshold is chosen to be the intensity value where the ratio of the number of pixels whose value is higher than the threshold to the total number of pixels in the image is closest to the given percentile. The P-tile method is one of the earliest thresholding methods based on the gray level histogram [4]. It assumes the objects in an image are brighter than the background, and occupy a fixed percentage of the picture area. This fixed percentage of picture area is also known as $\mathrm{P} \%$. The threshold is defined as the gray level that mostly corresponds to mapping at least $\mathrm{P} \%$ of the gray level into the object. Let $\mathrm{n}$ be the maximum gray level value, $\mathrm{H}$ (i) be the histogram of image $(\mathrm{i}=0 \ldots \mathrm{n})$, and $\mathrm{P}$ be the object area ratio. The algorithm of the P-tile method is as follows:

$$
\begin{gathered}
\mathrm{S} \leftarrow \text { sum }(\mathrm{H}(\mathrm{i})) \# \text { total image area \# } \\
\mathrm{F} \leftarrow \mathrm{S} \# \text { initialize all area as object area \# } \\
\text { For } \mathrm{k} \leftarrow 1 \text { to } \mathrm{n} \\
\mathrm{F} \leftarrow \mathrm{F}-\mathrm{H}(\mathrm{k}-1) \# \text { remove } \mathrm{k}-1 \text { from object area \# } \\
\text { If }(\mathrm{F} / \mathrm{t})<\mathrm{P} \text { then stop } \\
\mathrm{T} \leftarrow \mathrm{k} \# \text { final threshold value \# }
\end{gathered}
$$

This method is simple and suitable for all sizes of objects. It yields good anti-noise capabilities, however, it is obviously not applicable if the object area ratio is unknown or varies from picture to picture. Unfortunately, we do not usually have such definite information about the object area ratio. This information can sometimes be substituted by knowledge of another property, for example the average width of lines in drawings, shape, etc.

\section{Edge detection methods}

Edge detection is a fundamental tool used in most image processing applications to obtain information from images as a precursor step to feature extraction and object segmentation. This process detects boundaries between objects and the background in the image at which the image brightness changes sharply or more formally has discontinuities. The image containing these boundaries is known as edge map.

The purpose of detecting sharp changes in image brightness is to capture important events and changes in properties of the world there are many ways to perform edge detection , However most of them grouped into two categories, gradient and Laplacian. 
The gradient method detects the edges by looking for the local maximum and minimum in the first derivative of the image. The Laplacian method searches for zero crossings in the second derivative of the image. Some of the early gradient operators include Roberts [9], Prewitt [10], Sobel [11], Canny [12] edge operators. They involve small kernels to convolve with an image to estimate the first-order directional derivatives of the image brightness distribution. The edge value is calculated by forming a matrix centered on each pixel. If the value is larger than a given threshold, then the pixel is classified as an edge.

All the gradient-based algorithms have kernel operators that calculate the edge strength in directions which are orthogonal to each other, commonly vertically and horizontally. The contributions of the both components are combined to give the total value of the edge strength.

The Canny edge detection operator was developed by John F. Canny in 1986 and uses a multi-stage algorithm to detect a wide range of edges in images. It arises from the earlier work of Marr and Hildreth [13], who were concerned with modelling the early stages of human visual perception. His work is a gradient-based edge-finding algorithm that has become one of the most widely used edge detectors. This algorithm is known the optimal edge detector. In this situation, an "optimal" edge detector means following three criteria:

- Good detection: The algorithm should mark as many real edges in the image as possible.

- Good localization: Marked edges should be as close as possible to the edge in the real scene.

- Minimal response: A given edge in the image should only be marked once, and where possible, image noises should not create false edges. Based on these criteria, the canny edge detection process included the following stages:

- $\quad$ Noise removal: The canny edge detector smoothes the image to eliminate noise.

- Differentiation: It finds the image gradient in order to highlight regions with high spatial derivatives.

- $\quad$ Non-maximum suppression: The algorithm tracks along these already highlight regions and suppress any pixel that is not at the maximum.

Edge threshold, canny edge detector use a method called "hysteresis". The hysteresis method tracks along the remaining pixels that have not been suppressed. It uses two thresholds and if the gradient of the pixel is below the lower threshold, it is set to zero (regarded as a non-edge). If the gradient is above the higher threshold, it is set as an edge. If the gradient is between these thresholds, then it is set to zero unless there is a path from this pixel to a pixel with a gradient above the higher threshold. A widely used method for noise removal is the Gaussian filter, in which signals, in one and two dimensions, are smoothed out by the convolution of the image with a Gaussian kernel. The Gaussian operator is isotropic and therefore smoothes the image in all directions blurring sharp boundaries. All these approaches deal with the first derivatives of the image, thus slightly, but not totally, eliminate noises.

\section{New method}

The goal of new approach, Image Thresholding method utilize image characteristics to assist the thresholding process by combining modified P-tile algorithms as a global thresholding method with an edge detector to retrieve shape information. By using an edge detector, information of object area ratio acquired is determined by the shape of objects. This information is useful especially for applications that need to preserve the shape of objects in the original image.

Edge detectors are used to calculate the object area ratio by comparing the difference between edge map of the original image and edge map of the threshold image. By trying all of the possible object area ratio value to threshold an image and comparing each of their respective edge maps to the edge map of the original image, the best estimate of the object area ratio value is determined as the value where the produced edge map that has the smallest difference to that of original image. We employ the MSE (Mean Squared Error) to calculate the difference between edge map of the thresholded image and edge map of the original image. 
The figure 1 represents the diagram of different stages of our method. The algorithm is applied to several types of medical images; we present in figures 2 result segmentation with new method and compare with P-tile algorithm and Otsu's method on images.

This method is simple and suitable for all kind of edge detectors, since it only iterate in constant time (determine by Step value). It does not add anymore complexity to modified P-tile algorithms and the edge detector composing this new approach.

\section{Edge detector selection}

In the new method, we need to find the best edge detector to be combined with modified P-tile algorithms. We tried to combine the P-tile method with five kinds of edge detectors, Canny, Prewitt, Roberts, Sobel and Laplacian of Gaussian (LoG). The first four edge detectors are gradient based and the last one is Laplacian based.

We use the extraction of object from images. In more of images, shape information is needed to threshold images accurately. We employ 40 images, Some examples of the results are shown in Figures 2. According to the subjective evaluation of the results, we found that combining modified P-tile algorithms with canny edge detectors produce the most stable result. This combination consistently produces images

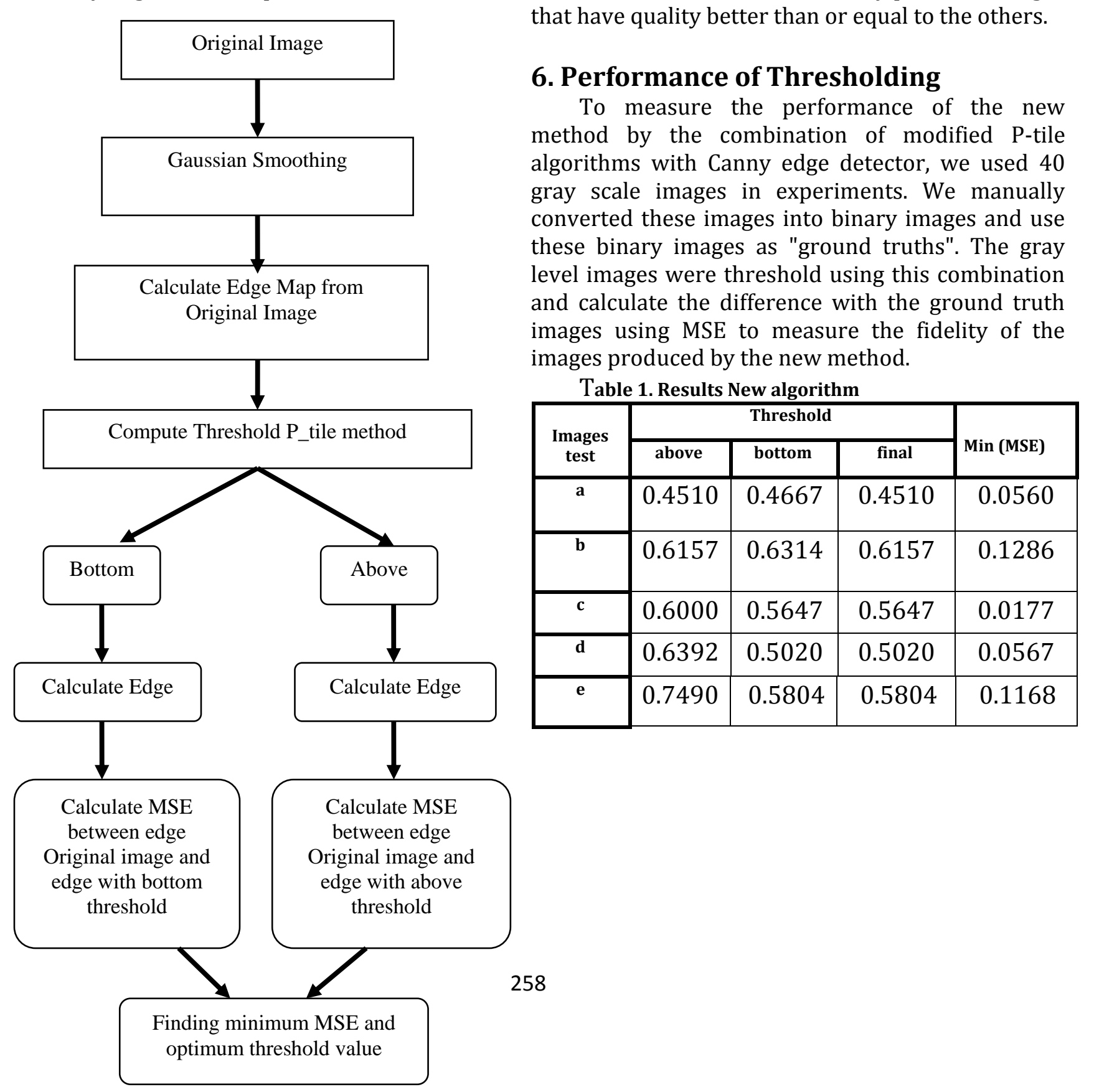




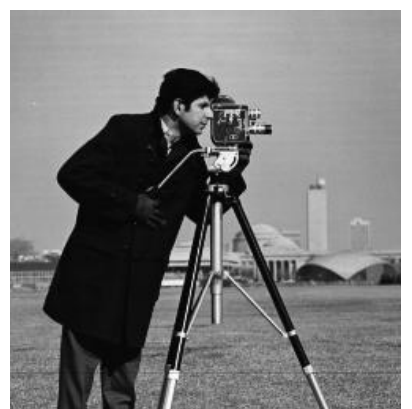

Original image

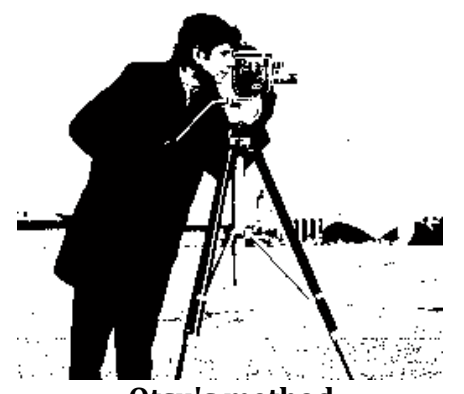

otsu's method
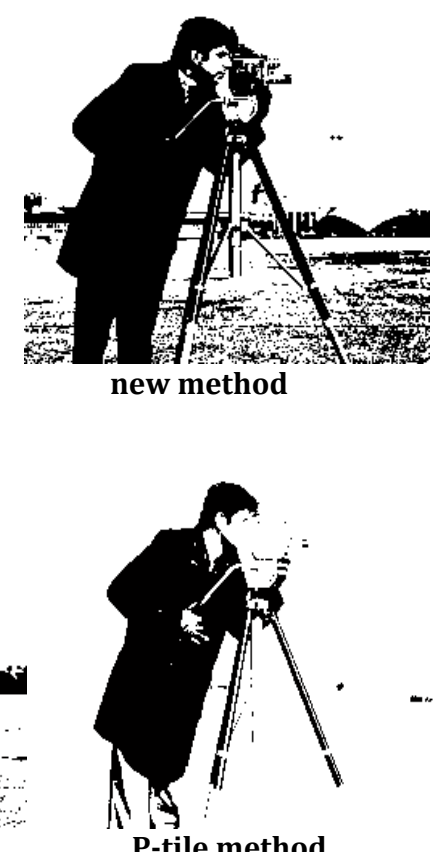

Figure 2a. Results "a” of test images

Figure 1. Segmentation Diagram new method

We also applied the same procedure using Otsu's method, P-tile method, which is well-known and used as one of the standards, for comparison. The result of this experiment is shown in Table 1.The result of this experiment shows that the new method is better than the Otsu method and P-tile methd in 38 out of 40 images.

\section{Conclusion}

We have proposed a new method of image thresholding by combining modified P-tile global thresholding algorithm and Canny edge detector. The Experimental results show that in average the performance of this method is significantly better than Otsu and P-tile methods.

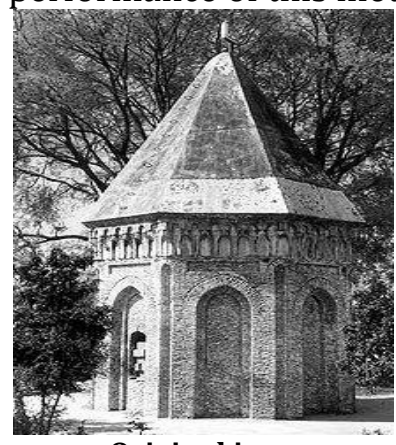

Original image

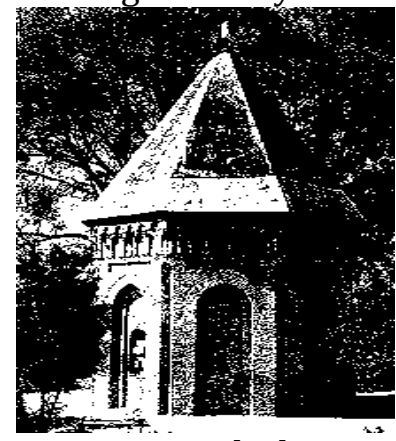

new method

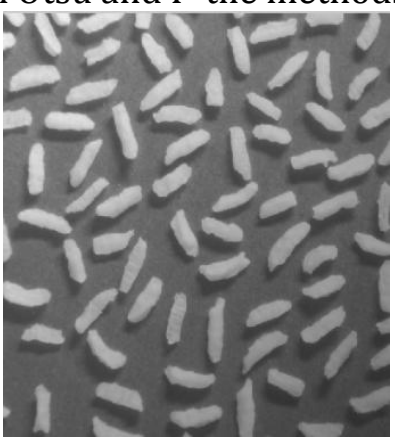

Original image

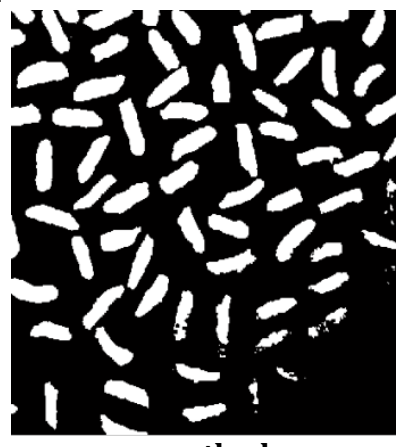

new method 

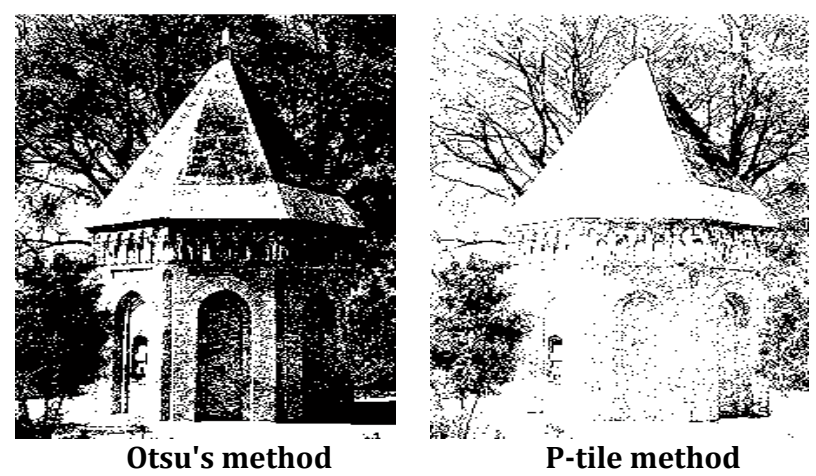

Figure 2b. Results “b” of test images

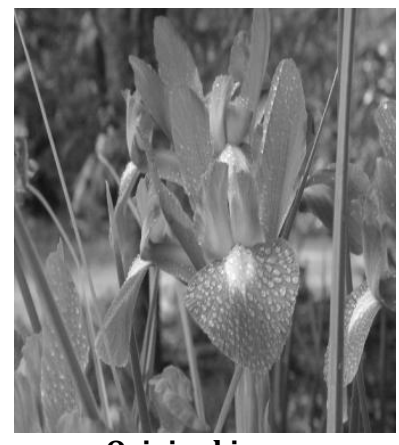

Original image

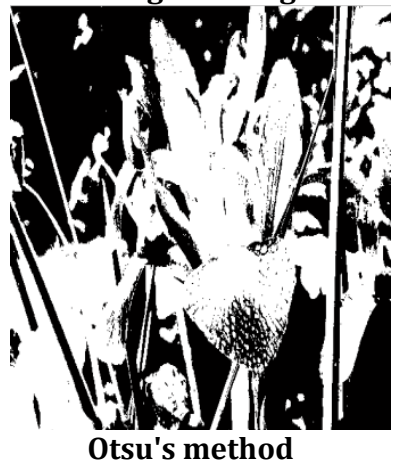

Figure 2d. Results "d" of test images

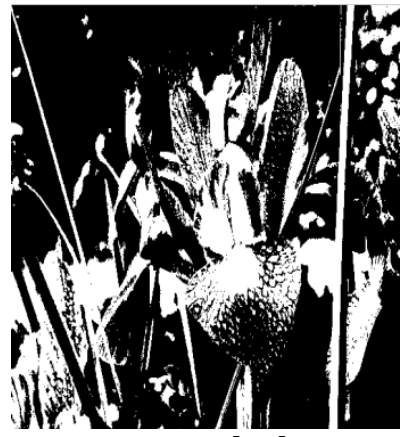

new method

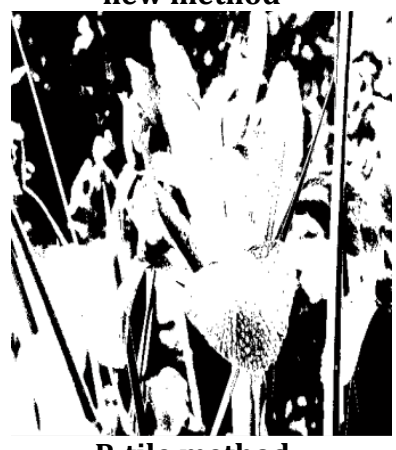

P-tile method

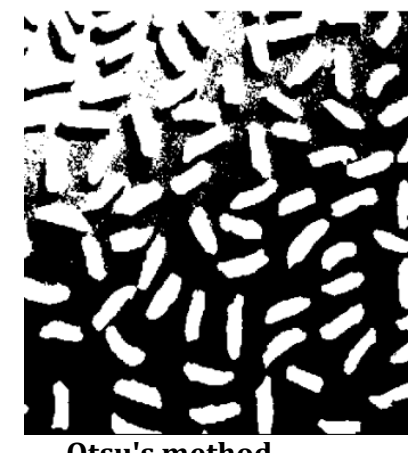

Otsu's method

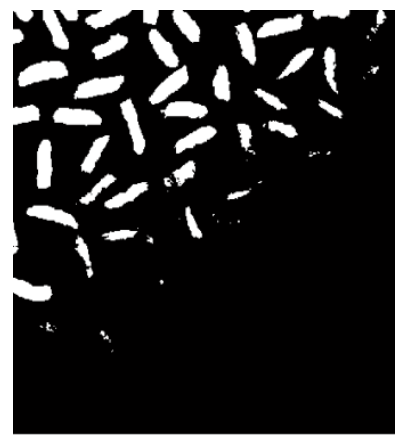

P-tile method

Figure 2c. Results "c" of test images

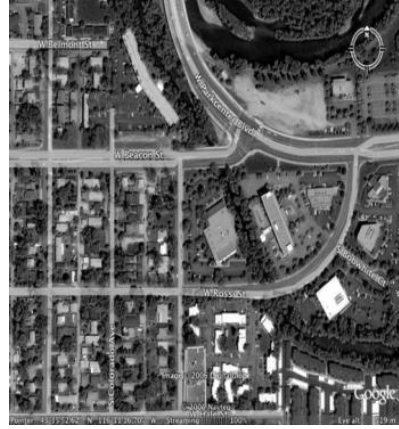

Original image

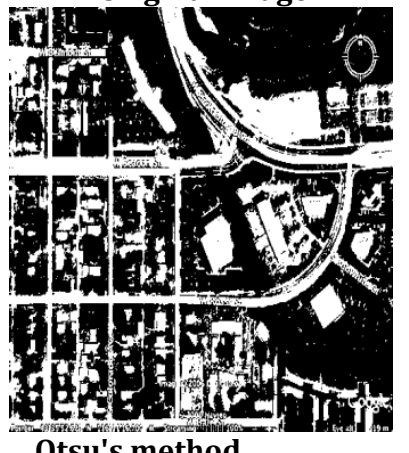

Otsu's method

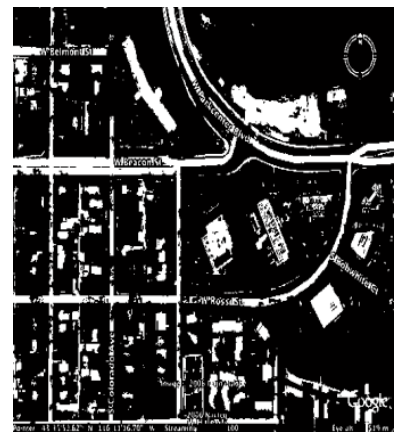

new method

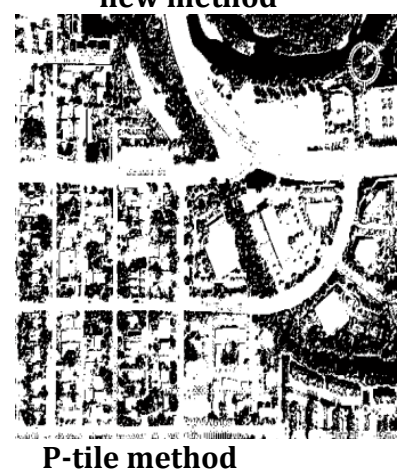

P-tile method

Figure 2e. Results "e" of test images

Figure 2. Results of test images

\section{References}

[1] A.S. Abutaleb, "Automatic Thresholding of Gray-Level Pictures Using Two Dimensional Entropy", Computer Vision, Graphics, and Image Processing, vol.47, pp.22-32,1989.

[2 J. Kittler and J. Illingworth, "Minimum Error Thresholding", Pattern Recognition, vol.19, no.1, pp.41-47, 1986.

[3] K.H. Liang and J.J.W Mao, "Image Thresholding by Minimizing the Measures of Fuzziness", Pattern Recognition, vol.28, no.1, pp.41-51, 1995.

[4] W. Doyle, "Operation useful for similarity-invariant pattern recognition", J. Assoc. Comput. Mach, vol.9, pp.259-267, 1962. 
[5] A.D. Brink and N.E. Pendock, "Minimum Cross-Entropy Threshold Selection", Pattern Recognition, vol.29, no.1, pp.179-188, 1996.

[6] J.N. Kapur, P.K. Sahoo, and A.K.C. Wong, "A New Method for Gray-level Picture Thresholding Using the Entropy of the Histogram", Computer Vision, Graphics, and Image Processing, vol.29, pp.273-285, 1985.

[7] N.R. Pal and S.K. Pal, "Entropic Thresholding", Signal Processing, vol.16, pp.97-108, 1989.

[8] S.K. Pal and A. Rosenfeld, "Image Enhancement and Thresholding by Optimization of Fuzzy Compactness",Pattern Recognition Letters, vol.7, pp.77-86, 1988.

[9] L. G. Roberts, "Machine perception of three-dimensional solids", in Optical and ElectroOptical Information Processing, J. T. Tippet et al., MIT Press, Cambridge, MA, pp. 159-197, 1965.

[10] J.M.S. Prewitt, "Object Enhancement and extraction", in Picture Analysis and Psychopictorics B.S. Lipkin and A. Rosenfeld (Eds.), Academic Press, New York, 1970.

[11] I. Sobel, "Neighbourhood coding of Binary images for test contour following and general array binary Processing", Computer Graphics Image Process, pp.127-135, 1975.

[12] J. F. Canny, "A computational approach to edge detection", IEEE Trans. Pattern Analysis and Machine Intelligence, vol.8, no.6, pp.679-698, 1986.

[13] D. Marr and E.C. Hildreth, "Theory of edge detection", Proc. Roy. Soc. London, B207, pp.187-217, 1980. 